\title{
Synchronization and tracking control of a novel 3 dimensional chaotic system
}

Basil H. Jasim*, Mofeed Turky Rashid, Khulood Moosa Omran

Electrical Engineering Department, University of Basrah, Basrah, Iraq

\author{
Correspondence \\ * Basil H. Jasim \\ Electrical Engineering Department, \\ University of Basrah, Basrah, Iraq \\ Email: hanbas632@gmail.com
}

\begin{abstract}
In this article, a novel three dimensional chaotic systems is presented. An extensive analysis including Lyapunov exponents, dissipation, symmetry, rest points with their properties is introduced. An adaptive tracking control system for the proposed chaos system has been designed. Also, synchronization system for two identical systems has been designed. The simulation results showed the effectiveness of the designed tracking and synchronization control systems.
\end{abstract}

KEYWORDS: Chaotic, dissipation, Lyapunov exponents, Synchronization.

\section{INTRODUCTION}

chaotic motion of dynamical systems is a special behavior arises in nonlinear systems, furthermore these systems are very sensitive to the initial conditions [1]. Chaotic systems received considerable attention in last three decades due to possible applications in varies science and engineering fields [2-5]. At other hand, chaos phenomena was investigated in many real systems [6], such as in double and triple pendulums [7,8] and brushless DC motor [9]. This leads to the fact that studying chaos systems and investigating their dynamical properties beside designing control systems for them are very advantageous and may be critical in some cases.

Lyapunov exponents represent sufficient indication on existence of chaotic behavior [10], where the system is chaotic when it has positive Lyapunov exponent. Systems with more than one positive exponent is said to be hyperchaotic [11].

Despite of that there was many chaotic and hyperchaotic systems proposed [12-15] since the first appear of chaotic systems by Lorenz [16], but it still benefits to find and analyze new chaotic systems for both theoretical and practical aspects [17].

Chaotic synchronization means using two identical chaotic systems, the first one called the master and the second is the slave. The two systems are synchronized where the controlled (slave) system should track the uncontrolled (master) system outputs. Due to complex dynamics of chaotic system, chaotic synchronization still a challenging problem [18]. Different control techniques have been used for chaotic synchronization system design [18-20].

In this work, a novel three dimensional chaos system is introduced. The proposed system has 5 terms with 2 quadratic nonlinearities. The system has been analyzed where its properties have been investigated. Lyapunov exponents have been found and from which and phase portrait, the system has been proved to be chaotic. Symmetry, dissipation, rest points and Kaplan York fractal dimension of the system have been found and discussed.

A tracking control system for all states of system assuming uncertain parameter values is designed. The design process uses simple algorithm with Lyapunov theory to find adaptive laws to estimate the uncertain parameters of the system. Also, and by using similar algorithm, a synchronization system has been designed to synchronize two identical systems.

The proposed system and the designed controllers have been simulated using Matlab. The simulation showed the effectiveness of the designed control systems.

The rest of this article is organized as follows: in section 2, the new chaotic system is introduced. In section 3 , the system dynamical properties are investigated. In section 4 , a tracking control system for the system is designed. In section 5, a synchronization controller for two identical systems is designed. Section 6 is a simulation study where Matlab18a has been used to write simulation programs for the control and synchronization systems. Finally, in section 7 the paper has been concluded.

\section{THE PROPOSED SYSTEM}

The proposed three dimensional chaos system is described by the following sate equations: -

$$
\left.\begin{array}{c}
\dot{x}_{1}=a x_{1} x_{2} \\
\dot{x}_{2}=70-x_{1} x_{3} \\
\dot{x}_{3}=b x-c x_{3_{1}}
\end{array}\right\}
$$


This system exhibits chaotic behavior for wide range of values of the parameters $a, b$ and $c$. we selected $a, b, c$ equal to $10,0.2$ and 0.6 respectively. The system has been simulated for initial conditions $\mathrm{x}_{1}, \mathrm{x}_{2}$ and $\mathrm{x}_{3}=\{0.2,0.2,0.2\}$. Figures below show phase portrait of the system. Figs. 1 and 2 show the 2 dimensional phase portrait of $\mathrm{x}_{1}, \mathrm{x}_{2}$ and $\mathrm{x}_{1}, \mathrm{x}_{3}$ planes and fig. 3 shows the 3 dimensional portrait of $x_{1}, x_{2}, x_{3}$.

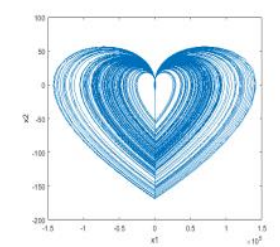

Fig. 1: 2 D phase portrait of $\mathrm{x}_{1}, \mathrm{x}_{2}$.

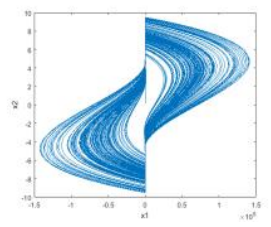

Fig. 2: phase portrait of $\mathrm{x}_{1}, \mathrm{x}_{2}$.

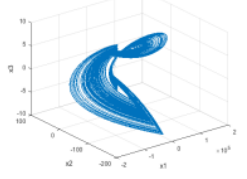

Fig. 3: phase portrait of $\mathrm{x}_{1}, \mathrm{x}_{2}, \mathrm{x}_{3}$.

\section{DYNAMICAL ANALYSIS}

\section{A. The Lyapunov exponents}

The Lyapunov exponents for the proposed system with the selected parameter values have been found using Wolf algorithm [21] and $X(0)=0.5,0.5,0.5$. The Lyapanove exponents dynamics for 1000 seconds are shown in fig. 4, and their steady state values are $\mathrm{L} 1=1.1, \mathrm{~L} 2=-0.37$ and L3=1.3324. the maximum exponent is positive which indicate clearly that the system is chaotic.

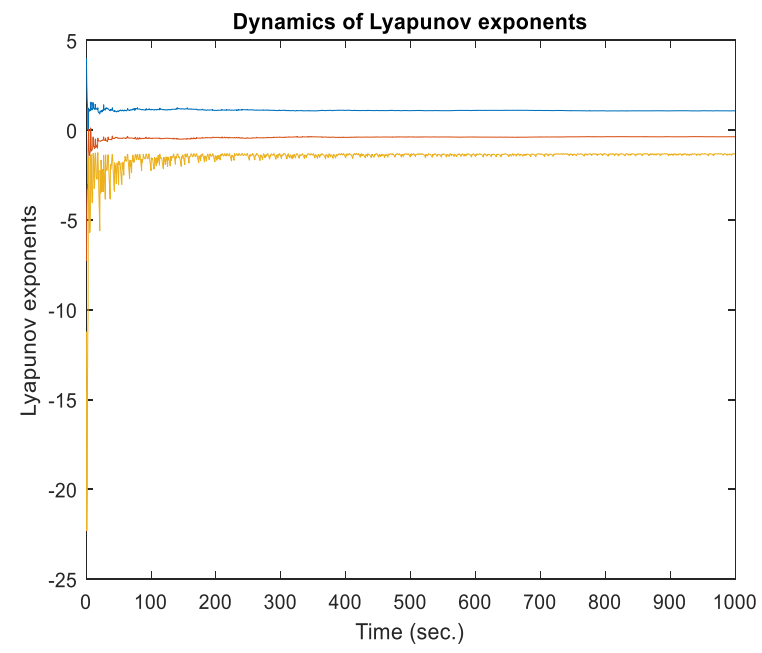

Fig. 4: Lyapunov exponents dynamics.

The Kaplan-York fractal dimension which can be used as a measure of system complexity is determined as follow :

The Lyapunov dimension which used as an indication about the degree of chaotic behavior of the system, can be found by Kaplan-York conjecture [23]. Using this formula, the following can be obtained:

$$
D_{K Y}=2+\frac{L 1+L 2}{|L 3|}=2.5479
$$

$D_{K Y}$ is The Lyapunov dimension.

\section{B. Dissipation}

Let us express the proposed system as a vector function $\mathrm{f}(\mathrm{x})$ :

$$
f(x)=\left[\begin{array}{c}
a x_{1} x_{2} \\
70-x_{1} x_{3} \\
b x_{1}-c x_{3}
\end{array}\right]
$$

The divergence of the system described by $f(x)$ can be found as in the following:

$$
\nabla . f=\sum_{i=1}^{3} \frac{\partial f_{i}}{\partial x_{i}}=-c=-0.6
$$

Since $\nabla . f=-0.6<0$, then the system is dispative because

$$
\dot{V}(t)=(\nabla \cdot f) V(t)=-0.6 V(t)
$$

Then, any volume element $\mathrm{V}(\mathrm{t})$ will shrink to 0 as $\mathrm{t}$ goes to zero. 


\section{The Equilibrium points}

The equilibrium or rest points can be found by setting the system questions equal to zero, i.e.

$$
\left.\begin{array}{ll}
a x_{1} x_{2}=0 & 1 \\
70-x_{1} x_{3}=0 & 2 \\
b x_{1}-c x_{3}=0 & 3
\end{array}\right\}
$$

In solving these questions, first we notice from 6-1 that either $\mathrm{x}_{1}=0$ or $\mathrm{x}_{2}=0$, but from $6-2, \mathrm{x}_{1}$ can not equal to zero, then $\mathrm{x}_{2}=0$. Solving 6-2 and 6-3, we find $x_{1}=$ ${ }_{ \pm} 14.4914$ and $x_{3}={ }_{-}^{+} 4.8305$. Then, the system has two equilibrium points $\left(\mathrm{E}_{1}\right.$ and $\left.\mathrm{E}_{2}\right)$ :-

$E_{1}=\left[\begin{array}{c}14.4914 \\ 0 \\ 4.8305\end{array}\right], \quad E_{1}=\left[\begin{array}{c}-14.4914 \\ 0 \\ -4.8305\end{array}\right]$

The general Jacobian matrix of the system with the specified parameters is given by the following: -

$$
J(x)=\left[\begin{array}{ccc}
20 x_{2} & 20 x_{1} & 0 \\
-x_{3} & 0 & -x_{1} \\
0.2 & 0 & 0.6
\end{array}\right]
$$

Using this matrix, we can find the spectral values of $E_{1}$ and $E_{2}\left(J\left(E_{1}\right)\right.$ and $\left.J\left(E_{2}\right)\right)$. The spectral values for both $E_{1}$ and $E_{2}$ are the same: -

$$
\lambda_{1,2}=3_{-}^{+} j 37.4156, \lambda_{3}=0
$$

It is clear that $E_{1}$ and $E_{2}$ are non-hyperbolic points. Then, the stability of these points cannot be ensured by linearization and the system can be self-excitation or hidden attractor.

\section{CONTROLLER DESIGN}

In this section, a tracking controller is designed using simple procedure. Rewriting the system model as: -

$$
\left.\begin{array}{c}
\dot{x}_{1}=a x_{1} x_{2}+u 1 \\
\dot{x}_{2}=70-x_{1} x_{3}+u 2 \\
\dot{x}_{3}=b x_{1}-c x_{3}+u 3
\end{array}\right\}
$$

Where $\mathrm{U}=\{\mathrm{u} 1, \mathrm{u} 2, \mathrm{u} 3\}$ is the control inputs. The design procedure based on satisfying the following error question: -

$$
\dot{e}_{\imath}+k_{i} e_{i}=0, \quad \mathrm{i}=1,2,3
$$

Where, $\mathrm{e}_{\mathrm{i}}=\mathrm{r}_{\mathrm{i}}-\mathrm{x}_{\mathrm{i}}, \mathrm{r}_{\mathrm{i}}$ is the desired state outputs. $\mathrm{k}_{\mathrm{i}}$ is the design parameters.

Combining (8) and (9) and solving for $\mathrm{U}$, the following results can be obtained;

$$
\left.\begin{array}{c}
u 1=\dot{r}_{1}+k_{1} e_{1}-a(t) x_{1} x_{2} \\
u 2=\dot{r}_{2}+k_{2} e_{2}-70+x_{1} x_{3} \\
u 3=\dot{r}_{3}+k_{3} e_{3}-b(t) x_{1}+c(t) x_{3}
\end{array}\right\}
$$

Substituting (10) into (8), the following equations are obtained: -

$$
\left.\begin{array}{c}
\dot{x}_{1}=\dot{r}_{1}+k_{1} e_{1}+(a-a(t)) x_{1} x_{2} \\
\dot{x}_{2}=\dot{r}_{2}+k_{2} e_{2} \\
\dot{r}_{3}+k_{3} e_{3}+(b-b(t)) x_{1}-(c-c(t)) x_{3}
\end{array}\right\}
$$

Substituting $\mathrm{x}_{\mathrm{i}}=\mathrm{r}_{\mathrm{i}}-\mathrm{e}_{\mathrm{i}}$ and $\dot{x}_{i}=\dot{r}_{i}-\dot{e}_{l}$ into (11), the error dynamics of the system can be written as in the following questions :-

$$
\left.\begin{array}{c}
\dot{e}_{1}=e_{a} e_{1} e_{2}-e_{a} e_{1} r_{2}-e_{a} e_{2} r_{1}+e_{a} r_{1} r_{2}-k_{1} e_{1} \\
\dot{e}_{2}=-k_{2} e_{2} \\
\dot{e}_{3}=e_{b} r_{1}-e_{b} e_{1}+e_{c} r_{3}+e_{c} e_{3}-k_{3} e_{3}
\end{array}\right\}
$$

Where, $e_{a}=a-a(t), e_{b}=b-b(t)$ and $e_{c}=c-c(t)$.

To stabilize the error dynamics of the system, we must obtain suitable update laws for $a(t), b(t)$ and $c(t)$ which ensures convergence to real values. For this purpose, Lyapunov theory has been used.

The following positive definite function is selected as a Lyapunov function candidate:

$$
V(t)=1 / 2\left(e_{1}^{2}+e_{2}^{2}+e_{3}^{2}+e_{a}^{2}+e_{b}^{2}+e_{c}^{2}\right)
$$

Differentiating V(t), we obtain: -

$$
\dot{V}(t)=e_{1} \dot{e}_{1}+e_{2} \dot{e_{2}}+e_{3} \dot{e_{3}}+\dot{a} e_{a}+\dot{b} e_{b}+\dot{c} e_{c}
$$

Substituting (12) into (14) and rearranging, the following result is obtained :-

$$
\dot{V}(t)=-k_{1} e_{1}^{2}-k_{1} e_{2}^{2}-k_{1} e_{3}^{2}+e_{a}\left(\dot{a}+e_{1}^{2} e_{2}-e_{1}^{2} r_{2}-\right.
$$

$\left.e_{1} e_{2} r_{1}+e_{1} r_{1} r_{2}\right)+e_{b}\left(\dot{b}+r_{1} e_{3}+e_{1} e_{3}\right)+e_{c}\left(\dot{c}+e_{3}^{2}-\right.$ $\left.r_{3} e_{3}\right)$

Selecting

$$
\left.\begin{array}{c}
\dot{a}=e_{1}^{2} e_{2}-e_{1}^{2} r_{2}-e_{1} e_{2} r_{1}+e_{1} r_{1} r_{2} \\
\dot{b}=r_{1} e_{3}+e_{1} e_{3} \\
\dot{c}=e_{3}^{2}-r_{3} e_{3}
\end{array}\right\}
$$

$\dot{V}(t)$ becomes: -

$$
\dot{V}(t)=-k_{1} e_{1}^{2}-k_{1} e_{2}^{2}-k_{1} e_{3}^{2}
$$

(17) is negative definite and controller stability is ensured.

\section{V.SYNCHRONIZATION SYSTEM DESIGN}

In this section, a synchronization system for two identical of the proposed chaotic system is designed. The master is the uncontrolled system described by (1). The slave system takes the following form: -

$$
\left.\begin{array}{c}
\dot{y}_{1}=a y_{1} y_{2}+u 1 \\
\dot{y}_{2}=70-y_{1} y_{3}+u 2 \\
\dot{y}_{3}=b y_{1}-c y_{3}+u 3
\end{array}\right\}
$$


Where $U=\{u 1, u 2, u 3\}$ is the control inputs to be designed to synchronize the two systems.

The error between the two systems (synchronization error) is defined as: -

$$
e_{i}=y_{i}-x_{i}, \quad i=1,2,3
$$

Using $\dot{e}_{i}=\dot{y}_{i}-\dot{x}_{i}$ and substuting (1) and (18), the following error dynamics can be easily obtained :-

$$
\left.\begin{array}{c}
\dot{e}_{1}=a\left(y_{1} y_{2}-x_{1} x_{2}\right)+u 1 \\
\dot{e}_{2}=x_{1} x_{3}-y_{1} y_{3}+u 2 \\
\dot{e}_{3}=b e_{1}-c e_{3}+u 3
\end{array}\right\}
$$

To stabilize the dynamics described by (20), we designed the control inputs to satisfy the following stable dynamics: -

$$
\dot{e}_{i}+k_{i} e_{i}=0, \quad \mathrm{i}=1,2,3
$$

Where $\mathrm{k}_{\mathrm{i}}$ is the design parameters.

Substituting (21) into (20) and solving for U, the following result is obtained: -

$$
\left.\begin{array}{c}
u 1=-k_{1} e_{1}-a(t)\left(y_{1} y_{2}-x_{1} x_{2}\right) \\
u 2=-k_{2} e_{2}-x_{1} x_{3}+y_{1} y_{3} \\
u 3=-k_{3} e_{3}-b(t) e_{1}+c(t) e_{3}
\end{array}\right\}
$$

Substituting (22) into (20), we get:-

$$
\left.\begin{array}{c}
\dot{e}_{1}=-k_{1} e_{1}+e_{a}\left(y_{1} y_{2}-x_{1} x_{2}\right) \\
\dot{e}_{2}=-k_{2} e_{2} \\
\dot{e}_{3}=-k_{3} e_{3}+e_{b} e_{1}-e_{c} e_{c}
\end{array}\right\}
$$

Where, $e_{a}=a-a(t), e_{b}=b-b(t), e_{c}=c-c(t)$.

To obtain stable update laws for time varying design parameters, we use Lyapunov theory. Selecting the Lyapunov function candidate as: -

$$
V(t)=1 / 2\left(e_{1}^{2}+e_{2}^{2}+e_{3}^{2}+e_{a}^{2}+e_{b}^{2}+e_{c}^{2}\right)
$$

Differentiating V, the following is obtained:-

$$
\dot{V}(t)=e_{1} \dot{e_{1}}+e_{2} \dot{e_{2}}+e_{3} \dot{e_{3}}+\dot{a} e_{a}+\dot{b} e_{b}+\dot{c} e_{c}
$$

Substituting from (23), the following result is obtained: -

$$
\begin{gathered}
\dot{V}(t)=-k_{1} e_{1}^{2}-k_{1} e_{2}^{2}-k_{1} e_{3}^{2}+e_{a}\left(\dot{a}+e_{1}\left(y_{1} y_{2}-\right.\right. \\
\left.\left.x_{1} x_{2}\right)\right)+e_{b}\left(\dot{b}+e_{1} e_{3}\right)+e_{c}\left(\dot{c}-e_{2} e_{3}\right)
\end{gathered}
$$

Selecting:

$$
\left.\begin{array}{c}
\dot{a}=-e_{1}\left(y_{1} y_{2}-x_{1} x_{2}\right) \\
\dot{b}=-e_{1} e_{3} \\
\dot{c}=e_{2} e_{3}
\end{array}\right\}
$$

$$
\dot{V}(t)=-k_{1} e_{1}^{2}-k_{1} e_{2}^{2}-k_{1} e_{3}^{2}
$$

Which is a negative semi definite function.

\section{SimUlation STUDY}

In this section, the designed control and synchronization systems are tested by simulation. Matlab18a is used to write the simulation programs.

\section{A. Control system}

The designed controller is simulated for two cases. First, a stabilization ability is tested by assuming starting from an initial condition and the reference target is:

$R=\{0,0,0\}$.

The initial condition is assumed to be:

$X(0)=\{1,-2,3\}$

Fig.5 shows system states responses and it is clearly shows the high performance of the control system.
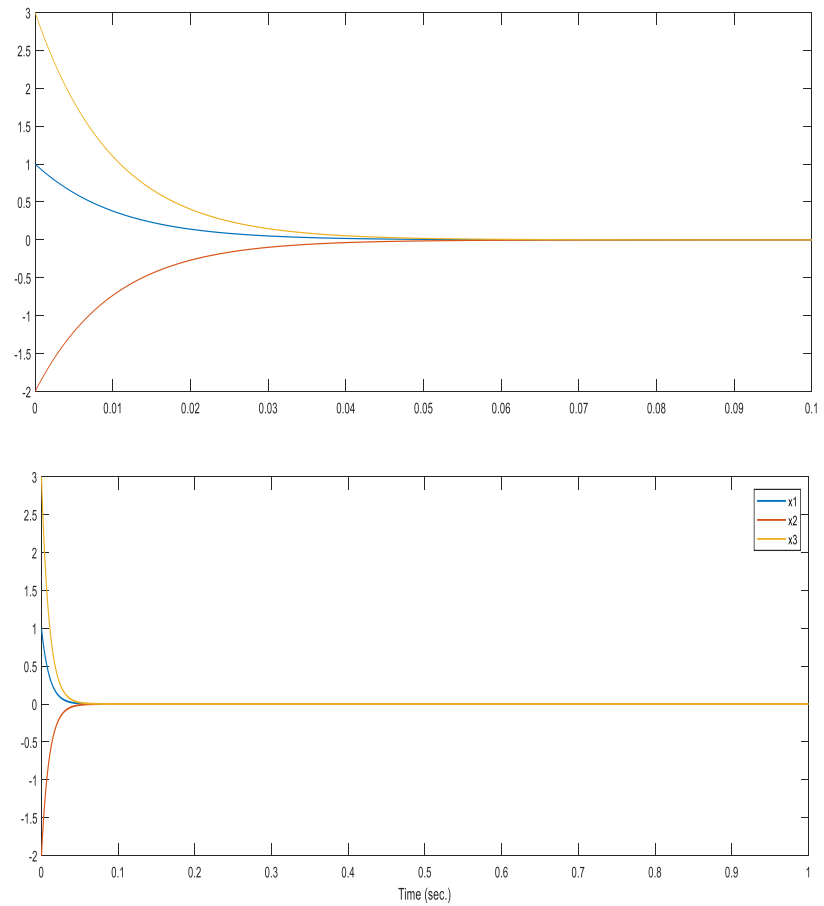

Fig. 5: States response of the stabilized system

Secondly, a tracking ability for the designed control system is tested by assuming sinusoidal reference signals and as follows:

$R=\{\sin (20 t), 2 \sin (20 t), 3 \sin (20 t)\}$

The initial condition is taken as:

$X(0)=\{1,-2,3\}$.

Fig. shows the states responses of the system and it shows high performance tracking ability.

Result in, 

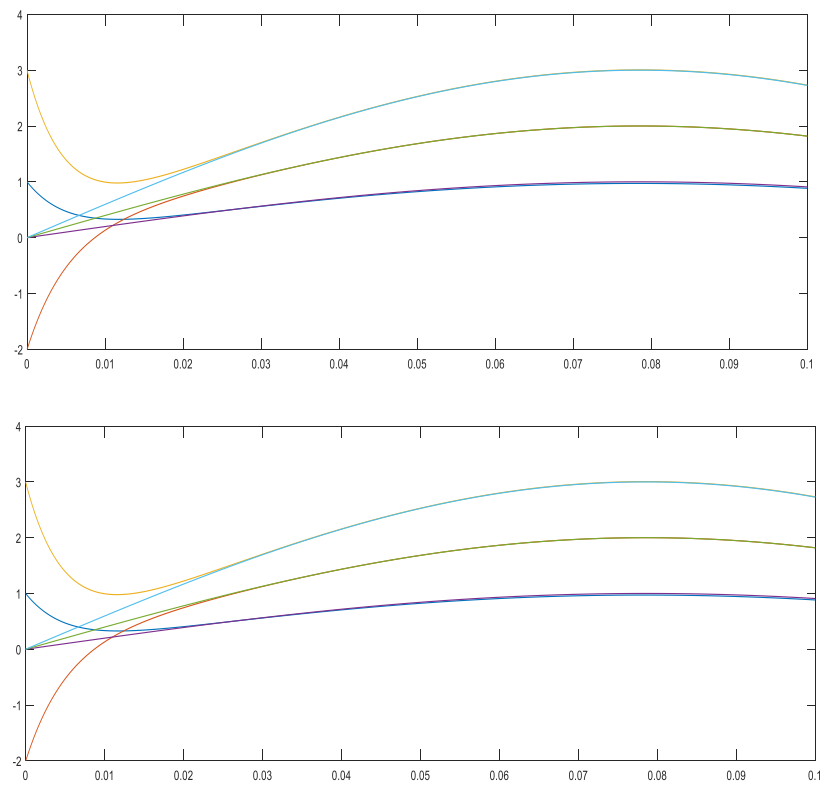

Fig. 6: States response of sinusoidal tracking system

\section{B. synchronization system}

The designed synchronization system is simulated assuming the following initial conditions for the master and slave states:

$X(0)=\{3,-1,2\}$

$Y(0)=\{1,2,3\}$

Fig.7 shows the synchronization errors and it clearly shows the high performance synchronization of the system with very small and acceptable transient time.

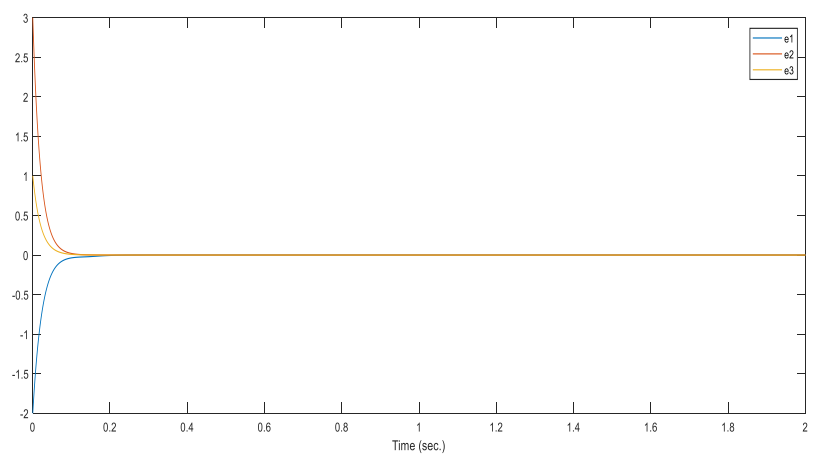

Fig. 7: Synchronization error.

The synchronization system is also simulated as a secure communication system where a sinusoidal signal $s=$ $3 \sin (20 t)$ is assumed as the signal to be transmitted. This signal is added to $x_{1}$ and the resultant signal $t_{r}$ is transmited to the receiver side where the slave system. The received signal should be extracted by subtracting it from $y_{1}$. The initial conditions for the master and slave are:

$X(0)=\{3,-1,2\}$

$Y(0)=\{1,2,3\}$

Fig. 8 shows the transmitted and received signals.
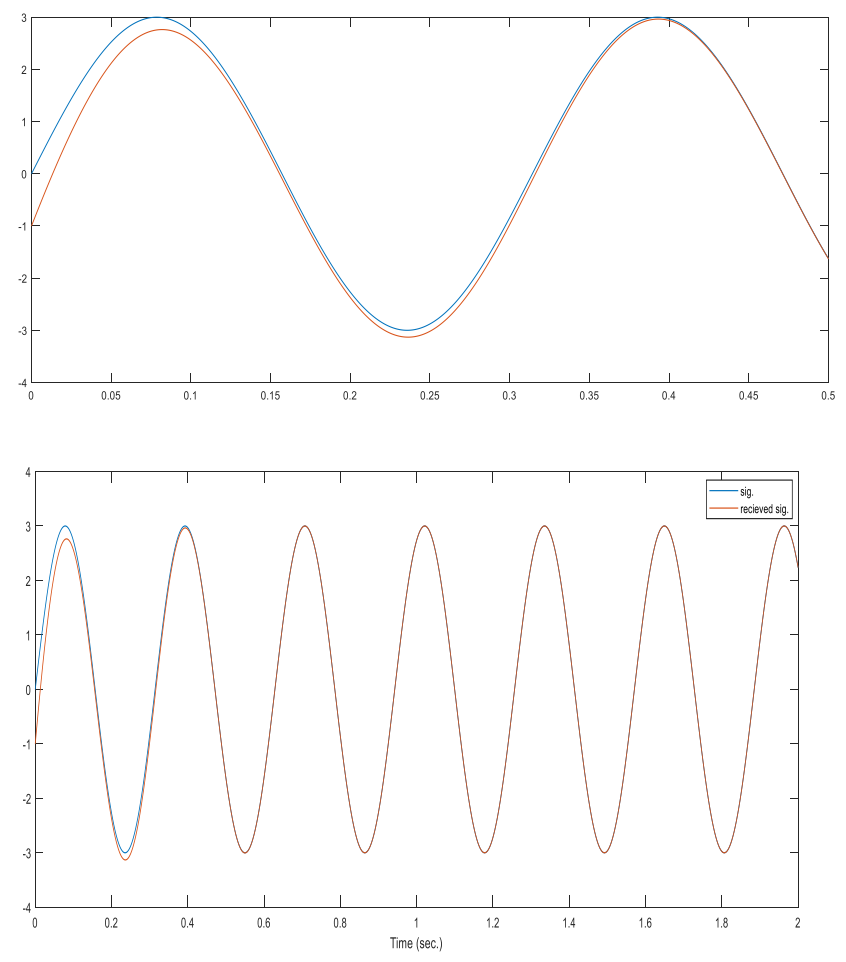

Fig. 8: Transmitted and received signal.

\section{CONCLUSION}

A novel 3 dimensional chaotic systems are presented in this paper. The dynamical properties of the proposed system are studied. Control and synchronization systems using simple control design procedure are designed. Simulation study is used to validate the designed control and synchronization systems and to investigate their performances. The simulation study shows that the controllers designed to control and synchronize the system have very high performances and that the synchronization system is suitable and easy to use in secure communication application.

\section{CONFLICT OF INTEREST}

The authors have no conflict of relevant interest to this article.

\section{REFERENCES}

[1] Ahmad Azar, Sundarapandian Vaidyanathan, (2015), "Chaos Modeling and Control Systems Design", 2015. DOI: 10.1007/978-3-319-13132-0.

[2] Saeed Khorashadizadeh, Mohammad-Hassan Majidi, "Chaos synchronization using the Fourier series expansion with application to secure communications", AEU - International Journal of Electronics and Communications, Vol. 82, 2017.

[3] Ghaida Al-Suhail, Fadhil Tahir, Mariam Abd, VietThanh Pham, Luigi Fortuna, "Modelling of Long-Wave Chaotic Radar System for Anti-Stealth Applications", 
Communications in Nonlinear Science and Numerical Simulation, Vol. 57, 2017.

[4] Amin Yousefpour, Hadi Jahanshahi, J.M. MunozPacheco, Stelios Bekiros, Zhouchao Wei, (2019), “A fractional-order hyper-chaotic economic system with transient chaos", Chaos Solitons \& Fractals, Vol. 130, 2019. DOI: 10.1016/j.chaos.2019.109400.

[5] Philippe Faradja, Guoyuan Qi, "Analysis of multistability, hidden chaos and transient chaos in brushless DC motor", Chaos Solitons \& Fractals, 2020. DOI: 132. 10.1016/j.chaos.2020.109606.

[6] Miillin T, "Chaos in physical systems. In: Crilly AJ, Earnshow RA, Jones H, edi- tors", Fractals and chaos. New York: Springer, 1991, pp. 237-245.

[7] Shinbrot T, Grebogi C, Wisdom J, Yorke JA. "Chaos in a double pendulum”, Am J Phys, Vol. 60, Issue 6, 1992.

[8] Zhu Q, Ishitobi M, "Experimental study of chaos in a driven triple pendulum", J Sound Vib, Vol. 227, Issue 1, 1999.

[9] Hemati N, "Strange attractors in brushless DC motors", IEEE Trans Circuits Syst, Vol. 41, Issue 1, 1994.

[10] Emad Mahmoud, Fatimah Abood, "A novel sort of adaptive complex synchronizations of two indistinguishable chaotic complex nonlinear models with uncertain parameters and its applications in secure communications", Results in Physics, Vol. 7, 2017. DOI: 10.1016/j.rinp.2017.07.050.

[11]Amin Yousefpour, Hadi Jahanshahi, J.M. MunozPacheco, Stelios Bekiros, Zhouchao Wei, "A fractionalorder hyper-chaotic economic system with transient chaos. Chaos Solitons \& Fractals, Vol. 130, 2019. DOI: 10.1016/j.chaos.2019.109400.

[12] Emad Mahmoud, "Dynamics and synchronization of new hyperchaotic complex Lorenz system", Mathematical and Computer Modelling, Vol. 55, 2012. DOI: 10.1016/j.mcm.2011.11.053.

[13] Maitreyee Dutta, Krishna Binoy, Roy, “A new fractional-order system displaying coexisting multiwing attractors; its synchronisation and circuit simulation", Chaos Solitons \& Fractals, Vol. 130, 2020. DOI: 109414. 10.1016/j.chaos.2019.109414.

[14] Mohammad Ababneh, "A new four-dimensional chaotic attractor", Ain Shams Engineering Journal, Vol. 9, 2016. DOI: 10.1016/j.asej.2016.08.020.

[15] Sundarapandian Vaidyanathan, Aceng Sambas, Mustafa Mamat, W. S. Mada Sanjaya, "A new three-dimensional chaotic system with a hidden attractor, circuit design and application in wireless mobile robot", Archives of Control Sciences, Vol. 27, 2017. DOI: 10.1515/acsc2017-0032.

[16]E.N. Lorenz, "Deterministic nonperiodic flow," J. Atmos. Phys., Vol. 20, pp 131-141, 1963.

[17] Mohammad Ababneh, "A new four-dimensional chaotic attractor. Ain Shams Engineering Journal, Vol. 9, 2017. DOI: 10.1016/j.asej.2016.08.020.
[18] Homayoon Arabyani, Hassan Saberi-Nik, "Synchronisation of the hyperchaotic complex Lorenz system in a finite time", International Journal of Modelling, Identification and Control, Vol. 25, 2016. DOI: 138. 10.1504/IJMIC.2016.075273.

[19] L.M. Pecora, T.L. Carroll, "Synchronization in chaotic systems", Phys. Rev. Lett., Vol. 64, pp 821-824, 1990.

[20] Mengjiao Wang, Xiaohan Liao, Yong Deng, Li Zhijun, Yongxin Su, Yicheng Zeng, "Dynamics, synchronization and circuit implementation of a simple fractional-order chaotic system with hidden attractors", Chaos, Solitons \& Fractals, Vol. 130, 2020. DOI: 109406. 10.1016/j.chaos.2019.109406.

[21] Wolf A, Swift J, Swinney H, Vastano J., "Determing Lyapunov exponents from a time series", Phys Lett B, Vol. 737, 1985.

[22] P. Frederickson, J.L. Kaplan, E.D. Yorke, J.A. Yorke, "The Liapunov dimension of strange attractors", J. Differ. Equ., Vol. 44, pp. 185-207, 1983. 